\title{
Population structure of Cylindrocladium parasiticum infecting peanuts (Arachis hypogaea) in Georgia, USA
}

\author{
Louwrance P. Wright • Andrew J. Davis • \\ Brenda D. Wingfield • Pedro W. Crous • \\ Tim Brenneman • Michael J. Wingfield
}

Accepted: 11 January 2010

(C) KNPV 2010

\begin{abstract}
Cylindrocladium parasiticum is an important pathogen of peanut (Arachis hypogaea) causing the disease Cylindrocladium black rot. The genetic structure of this haploid pathogen was determined for populations associated with peanut in Georgia, USA. Ten polymorphic microsatellite markers were used to determine genetic and genotypic diversity among three sub-populations representing the geographic distribution of peanut production in Georgia. Among 200 isolates collected, only 10 unique multilocus haplotypes were identified, with one multilocus haplotype occurring 176 times. Although no evidence for random mating was observed, the existence of loops in a constructed haplotype network hint at the possibility of recombination events. The absence of random mating might therefore be attributed to the
\end{abstract}

A. J. Davis

Department of Biochemistry,

Max Planck Institute for Chemical Ecology,

Hans-Knoell-Strasse 8,

07745 Jena, Germany

L. P. Wright · B. D. Wingfield · M. J. Wingfield

Department of Genetics, Tree Protection Co-operative

Programme, Forestry and Agricultural Biotechnology

Institute (FABI), University of Pretoria,

Pretoria 0002, South Africa

P. W. Crous

Centraalbureau voor Schimmelcultures (CBS),

P.O. Box 85167, 3508 Ad Utrecht, The Netherlands homothallic nature of $C$. parasiticum as well as the clonality of the population, without excluding the possible occurrence of recombination. Cylindrocladium parasiticum thus appears to consist of a genetically homogeneous population throughout Georgia with mainly clonal reproduction or inbreeding contributing to the observed population genetic structure.

Keywords Calonectria ilicicola $\cdot$ Haplotype network · Homothallic $\cdot$ Microsatellites $\cdot$ Population genetics $\cdot$ Simple sequence repeat $(\mathrm{SSR})$

\section{Abbreviations \\ CBR Cylindrocladium black rot \\ MLH Multilocus haplotypes}

\author{
T. Brenneman \\ Department of Plant Pathology, CPES, \\ University of Georgia, \\ Tifton, GA 31794, USA
}

Present Address:

L. P. Wright $(\bowtie)$

Department of Biochemistry,

Max Planck Institute for Chemical Ecology,

Hans-Knoell-Strasse 8,

07745 Jena, Germany

e-mail: lwright@ice.mpg.de 
IMH Indistinguishable multilocus haplotypes

DMH Distinguishable multilocus haplotypes

\section{Introduction}

Cylindrocladium parasiticum [Crous, Wingfield \& Alfenas] $(=C$. crotalariae $)$ is a haploid fungus with a sexual state (teleomorph) referred to as Calonectria ilicicola ( $=$ C. crotalariae) (Crous et al. 1993). Cylindrocladium parasiticum is a soil-borne fungus and is an important pathogen of peanuts (Arachis hypogaea L.), causing the disease known as Cylindrocladium black rot (CBR). CBR was first recorded in 1965 in Georgia causing root, peg and pod rot of peanuts (Bell and Sobers 1966) and has since spread to all of the peanut producing areas in the southeastern USA. Since CBR does not occur regularly over large areas, losses are generally not as high as for other peanut diseases in Georgia. Where it occurs, however, it is often the largest factor limiting production (Brenneman 2003) with losses of up to $50 \%$ in yield (Bell et al. 1973).

The Calonectria ilicicola sexual state of C. parasiticum is commonly seen in the field when moisture levels are high. However, C. parasiticum is known to be homothallic (Alfieri et al. 1982) implying that these sexual structures may not reflect genetic recombination between distinct haplotypes. Nothing is known regarding the role of the sexual state in nature. Rowe and Beute (1975) found ascospores to be discharged in viscous ooze and to be extremely susceptible to lethal desiccation. They therefore concluded that ascospores would most probably be limited to short-distance, within-field spread.

Knowledge of the population structure of $C$. parasiticum might help to explain the evolutionary processes shaping and maintaining genetic variation within and among populations as has been shown in other fungi (Milgroom 1996; Milgroom and Peever 2003). An understanding of population structure could also help to determine the evolutionary potential of $C$. parasiticum to overcome genetic resistance (McDonald and Linde 2002), and to predict the efficacy of host plant resistance or fungicide treatments (Milgroom and Peever 2003). Population structure can, for example, indicate the potential durability of resistant cultivars which, in turn, helps in establishing a cultivar rotation schedule.

To further our understanding of the epidemiology and management of CBR we estimated genetic and genotypic diversities and tested for population differentiation among $C$. parasiticum sub-populations in Georgia, USA. This was achieved by sampling three sub-populations of isolates collected from single fields. Another group of isolates was collected from more distant sites in Georgia. Microsatellite markers previously developed for this pathogen (Wright et al. 2006) were used to estimate population genetic structure.

\section{Materials and methods}

\section{Samples}

Isolates of C. parasiticum were collected during 2001 from infected peanut fields in Georgia, USA. Where multiple samples were collected within a field, isolations were made from plants on random transects across the field. Sampling was conducted in 13 different counties and from 26 different sites. The isolates were subdivided into three different subpopulations (GA1, GA2 and GA3) coming from single sites and a fourth collection (GAMULT) comprising isolates from 23 different sites in 12 counties. GA1, with 33 isolates, and GA2, with 94, were collected from different sites $12 \mathrm{~km}$ apart in Burke county. The third sub-population (GA3, 31 isolates) was collected from Calhoun county, approximately $320 \mathrm{~km}$ away from GA1 and GA2. The GAMULT group of isolates from different sites included 42 isolates. The sub-populations GA1, GA2 and GA3 were each collected from single fields, whereas the GAMULT group of isolates were sampled on a broader scale from different sites throughout Georgia.

Isolation, cultures and DNA isolation

Isolates were collected after the peanuts were inverted and when the clusters of diseased plants were clearly visible. One sample was taken at each disease locus and, therefore, each isolate represents a unique infection event. Infected plant tissue was surface disinfected in $10 \%$ Clorox ( $5 \mathrm{ml}-1$ sodium hypochlorite) for 2 to 
3 min, rinsed with water and plated on potato-dextrose agar (PDA). Most isolations were made from infected pods, although some isolates were obtained from infected stems or kernels.

After isolation, all the samples were cultured on 20 gl-1 malt extract agar (MEA) and then stored by immersing blocks of MEA bearing mycelium in sterile water maintained in airtight McCartney bottles at room temperature. Before DNA extraction, these isolates were transferred to synthetic nutrient agar (SNA) and incubated for $1-2$ weeks at $22^{\circ} \mathrm{C}$ under near UV light before being transferred to MEA. After incubation at room temperature for 7-9 days, surface mycelium was removed and DNA was extracted using a modified protocol based on the method of Möller et al. (1992).

The mycelium was added to $600 \mu 1$ extraction buffer containing $100 \mathrm{mM}$ Tris-HCl ( $\mathrm{pH} 8.0$ ), $10 \mathrm{mM}$ EDTA, $2 \%$ SDS (w/v) and $100 \mu \mathrm{g}$ Proteinase $\mathrm{K}$ (Sigma) and homogenised with a glass rod. After a freeze-thaw cycle the mycelium suspension was incubated at $60^{\circ} \mathrm{C}$ for $60 \mathrm{~min}$, after which $180 \mu \mathrm{l}$ $5 \mathrm{M} \mathrm{NaCl}$ and $80 \mu 110 \%$ CTAB (w/v) were added. The suspension was then vortexed and incubated for a further $10 \mathrm{~min}$ at $65^{\circ} \mathrm{C}$. After adding $860 \mu \mathrm{l}$ chloroform:isoamylalcohol $(24: 1)(\mathrm{v} / \mathrm{v})$ and vortexing, the suspension was incubated on ice for $30 \mathrm{~min}$. The nonaqueous phase was then separated from the aqueous phase by centrifuging at max in a microcentrifuge and the DNA precipitated from the aqueous phase in a new microcentrifuge tube with 0.55 volumes isopropanol. After centrifuging for $5 \mathrm{~min}$ at $\max$ in a microcentrifuge, the DNA pellet was washed with $70 \%$ ethanol, dried, dissolved in $100 \mu \mathrm{l}$ of water and stored at $-20^{\circ} \mathrm{C}$.

Microsatellite markers

A set of 12 polymorphic microsatellite markers developed for C. parasiticum (Wright et al. 2006) was used to detect polymorphisms among the 200 isolates used in this study. Two of these markers (CypaL8 and CypaL18) were monomorphic in this population and were not included in the data analysis. The markers were amplified following Wright et al. (2006). The forward primers were labelled with either 6FAM, VIC, NED or PET 5'-fluorescent dye (Applied Biosystems Inc., Foster City, CA). After amplification GeneScan-500 LIZ Size Standard (Applied Biosystems) was added and the sizes of the PCR products were determined on an ABI PRISM 3100 Genetic Analyzer (Applied Biosystems) and analyzed using ABI PRISM GENEMAPPER version 3.0 analysis software (Applied Biosystems).

AFLP analysis

AFLP marker profiles were obtained for 9 isolates from sub-population GA3, 10 isolates from sub-population GA2 and 15 isolates from sub-populations GA1 and GAMULT.

The AFLP markers were developed using the method of Vos et al. (1995). Total genomic DNA (100 ng) was digested with $2 \mathrm{U}$ EcoRI and MseI respectively for $2 \mathrm{~h}$ at $37^{\circ} \mathrm{C}$, after which EcoRI (1 pmol) and $M s e \mathrm{I}(10 \mathrm{pmol})$ adaptors were ligated to the digested DNA at $37^{\circ} \mathrm{C}$ for $3 \mathrm{~h}$ using 1 Weiss U T4 DNA ligase (Roche). The final volume of the restriction/ligation reaction was $40 \mu \mathrm{l}$, of which $5 \mu \mathrm{l}$ was used as template in the first pre-amplification reaction. The primers used for the pre-amplification had sequences homologous to the EcoRI and MseI adaptors with no selective nucleotides at their 3 ' ends. The pre-amplification reaction conditions were $1.5 \mathrm{mM} \mathrm{MgCl} 2,3 \mu \mathrm{l} 10 \mathrm{X}$ PCR buffer and $1 \mathrm{U} \mathrm{Taq}$ polymerase (Roche), $0.2 \mathrm{mM}$ dNTP's, and $0.3 \mu \mathrm{M}$ of each primer, made up to a final volume of $30 \mu \mathrm{l}$ with water. The temperature profile was for $30 \mathrm{~s}$ at $72^{\circ} \mathrm{C}$ followed by 30 cycles of $30 \mathrm{~s}$ at $94^{\circ} \mathrm{C}, 30 \mathrm{~s}$ at $56^{\circ} \mathrm{C}$ and $60 \mathrm{~s}$ at $72^{\circ} \mathrm{C}$ with a final extension step of $120 \mathrm{~s}$ at $72^{\circ} \mathrm{C}$. After the first cycle, each elongation step was increased by one second. The PCR products were diluted 1:10, of which $5 \mu$ was used as the template in the final amplification using EcoRI and MseI primers with two adenosine selective nucleotides on their $3^{\prime}$ ends. The EcoRI primer was fluorescencelabelled with IRDye700 (Biolegio, Malden, The Netherlands). The PCR conditions were $0.5 \mathrm{mM}$ $\mathrm{MgCl}_{2}, 2 \mu \mathrm{l}$ 10X PCR buffer and $1 \mathrm{U}$ Taq polymerase (Roche), $0.2 \mathrm{mM}$ dNTP's, $0.2 \mu \mathrm{M}$ EcoRI primer and $0.3 \mu \mathrm{M} \mathrm{MseI}$ primer, and water to make a final volume of $20 \mu \mathrm{l}$. The temperature profile was $10 \mathrm{~s}$ at $94^{\circ} \mathrm{C}, 30 \mathrm{~s}$ at $65^{\circ} \mathrm{C}$ with a decrease of $0.7^{\circ} \mathrm{C}$ per cycle and $60 \mathrm{~s}$ at $72^{\circ} \mathrm{C}$ for the first 13 cycles. The last 23 cycles were done with a constant annealing temperature of $56^{\circ} \mathrm{C}$ and with a time elongation of one second per cycle. All PCR reactions were performed on a BioRad iCycler version 3.021 thermocycler. 
Formamide loading buffer $(10 \mu \mathrm{l})$ was added to the PCR products and heated at $90^{\circ} \mathrm{C}$ for $180 \mathrm{~s}$ and then snap-cooled on ice. The PCR products $(0.8 \mu \mathrm{l})$ were then analysed on $80 \mathrm{gl}-1$ denaturing polyacrylamide gels with 1.0 X TBE running buffer using a $4200 \mathrm{Li}-$ $\mathrm{COR}^{\circledR}$ automated DNA sequencer (LI-COR, Lincoln, NE, USA).

\section{Data analysis}

\section{Microsatellite analysis}

The microsatellite data were used to determine allele frequencies at each locus from the different subpopulations and standard population statistics were determined using the software POPGENE Version 1.32 (Yeh et al. 1999) and MULTILOCUS 1.3 (Agapow and Burt 2001). Genetic diversity was calculated for each locus as $H=1-p_{i}^{2}$, where $p_{i}$ is the frequency of the $i$ th allele at each locus, and averaged over all loci (Nei 1973). The genetic diversity $(H)$ represents the probability that two randomly sampled alleles will differ at a locus, with a value of one indicating that all alleles are different for the locus. Genotypic diversity was calculated using the method of Stoddart and Taylor (1988). When all isolates in the population have a different haplotype, the $\hat{G}$ value will be at its maximum and equal to the number of isolates. To compare the genotypic diversity between sub-populations with different sample sizes, a $t$-test was conducted by calculating the variances of the different $\hat{G}$ values (Stoddart and Taylor 1988).

Population differentiation was tested by comparing allele frequencies among the sub-populations using Weir \& Cockerham's $\theta$ value (Weir and Cockerham 1984). The $\theta$-value was estimated under the null hypothesis of non-differentiation among subpopulations, when $\theta=0$. Statistical analysis was done by comparing the calculated $\theta$-values to data sets in which isolates have been randomised across populations 10000 times using the program MULTILOCUS.

\section{Testing clone frequency}

There were only small numbers of isolates after clone correction and, therefore, the standard tests for random mating were not statistically significant. A method based on the loss of distinguishable multi- locus haplotypes (DMH) when only a subset of the original markers is used, was therefore used (Stoddart and Taylor 1988). The test is based on the fact that a randomly mating population will have significantly fewer indistinguishable multilocus haplotypes (IMH) after reduction in the number of markers. In such a population, there will be random recombination of alleles amongst isolates, independently for each locus. Therefore, the expectation is that each subset will contain a larger number of unique multilocus haplotypes and fewer IMH compared to a clonal population. If the population is clonal, a significantly larger number of IMH will emerge. By using a large enough number of subsets of randomly chosen markers, taking the precaution not to repeat the same subset of markers, the hypothesis of random mating can be statistically tested for a small number of multilocus haplotypes (MLH).

A very large amount of recombination and sex is simulated by randomising alleles among isolates independently for each locus. Statistical significance is then calculated by comparing the observed amount of IMH to that expected under the hypothesis of complete panmixis simulated by 100 randomisations. This number of randomisations was sufficient because the distribution of expected values stabilised before 100 randomisations were completed.

Random mating was tested for the total microsatellite data set containing $10 \mathrm{MLH}$ after clone correction. Using PopTools version 3.0 (Hood 2008), 100 random subsets of 5 each of the 10 possible microsatellite markers were produced (100 microsatellite marker subsets). The number of IMH occurring in each subset was then determined and the total amount of IMH for the 100 subsets were added up. Each IMH was counted as the event where DMH could not be distinguished in the subset. Thus, when 4 $\mathrm{DMH}$ distinguishable in the original data set (e.g. DMH A, B, C and D), could not be distinguished for a specified subset of 5 microsatellite markers, the number of IMH would be counted as 6 (because of the occurrence of IMH AB, AC, $\mathrm{AD}, \mathrm{BC}, \mathrm{BD}$ and $\mathrm{CD})$. The original data set was then randomised 100 times to simulate random mating, as described above. The total number of IMH for each of these simulated data sets was then determined using the 100 microsatellite marker subsets used to determine the IMH of the original data. The number of IMH in the original data set was then compared to the distribution of IMH 
numbers produced from the simulations of random mating. The statistical significance, $\mathrm{P}$, the one-tailed probability of obtaining the observed IMH or greater under the hypothesis of random mating, is then calculated as (area of distribution simulated $>$ observed IMH value)/(total area of distribution simulated).

The AFLP data were similarly tested for random mating, by producing 100 subsets of 10 AFLP markers each of the 25 possible polymorphic AFLP markers.

\section{Creating a haplotype network}

To reveal the interrelationships of different isolates in the GAMULT data set, we constructed the medianjoining, maximum parsimony, haplotype network using Network 4.5.1.0. (Fluxus Technology Ltd. 2008). This software identifies the shortest least complex phylogenetic network linking all the haplotypes (Bandelt et al. 1999).

\section{Results}

\section{Marker selection}

The 10 polymorphic microsatellite markers detected between two and four alleles per marker, with an average of 2.5 alleles per marker for the Georgia population. Computing genotypic diversity and plotting it against the number of loci using randomisation procedures in MULTILOCUS also revealed that 10 markers were sufficient to analyse population structure. Using this analysis showed that eight markers accounted for $92 \%$ of the observed genotypic diversity in the Georgia population. A single additional marker accounted for $96 \%$. Since it is not possible to force an outcross between two isolates that are polymorphic for the microsatellite markers, the linkage between the markers could not be tested.

To test that the clonality observed was not due to linkage of the microsatellite markers, a subset of 49 isolates were subjected to AFLP analysis. The AFLP profiles consisted of 116 markers, of which 25 were polymorphic, identifying 22 unique MLH.

Genetic and genotypic diversity

A total of 10 unique MLH were identified among the 200 isolates and 10 microsatellite markers analysed in this study. The Georgia populations were highly clonal, with 176 isolates having a single haplotype, and two other haplotypes occurring in at least two sub-populations. The single most common haplotype thus accounted for $88 \%$ of the isolates from Georgia. The most common haplotype in the three subpopulations was also the predominant haplotype in the GAMULT group of isolates. The genotypic diversities for the sub-populations from Georgia ranged between 1.067 and 1.592 (Table 1). At a significance level of $95 \%$, there were no differences between the genotypic diversities of the different subpopulations $(0.455<P<0.997)$. The Georgia subpopulations also had low genetic diversities ranging from 0.054 to 0.094 (Table 1).

\section{Population sub-division}

No significant overall population differentiation was found among the three sub-populations in Georgia based on Weir and Cockerham's (1984) population differentiation statistic $\theta(\theta=0.018, P=0.117)$. There were also no significant differences between the individual sub-populations with $\theta$ of $0.008-0.051$ and $\mathrm{P}$ of $0.248-0.058$. The greatest population differentiation was between GA1 and GA3 $(\theta=$ $0.051)$, but this was not significantly different from zero $(P=0.058)$.

\section{Random mating}

Random mating was tested with a method based upon the reduction in the number of DMH when only a subset of markers is used to distinguish between isolates. Because the number of unique MLH observed for the microsatellite data was very low, and since there was no evidence of population differentiation, the complete data sets were used for random mating testing. The microsatellite data had an IMH number of 774, with the distribution of the IMH values for the simulated random mating datasets of 269-625. The observed IMH value for the AFLP data was 3142 with the values for the simulated data sets ranging from 1759-2831. The observed value for the number of IMH derived from the microsatellite data was thus very significantly higher than the expectation under random mating (1-tailed $P<<<0.0001$, by simulation), clearly showing no evidence of random mating. The same was true for the observed value 
Table 1 Microsatellite analysis to determine the genotypic and genetic diversities, and the occurrence of multilocus haplotypes (MLH) among three sub-populations, the GAMULT group of isolates and the entire sampled population of Cylindrocladium parasiticum isolated from peanuts in Georgia

\begin{tabular}{lccccrr}
\hline & \multicolumn{2}{l}{ Population or group of isolates } & & \\
\cline { 2 - 7 } MLH & MLH no. & GAMULT & GA1 & GA2 & GA3 & GATOTAL \\
\hline BBABBBDACA & 1 & 33 & 27 & 86 & 30 & 176 \\
BAACBBDACB & 2 & 0 & 0 & 2 & 0 & 2 \\
BAACBBDABB & 3 & 3 & 4 & 5 & 0 & 12 \\
BBABBBCACA & 4 & 1 & 0 & 0 & 0 & 1 \\
BBABBBAACA & 5 & 2 & 0 & 0 & 0 & 2 \\
BBABBBDACB & 6 & 2 & 1 & 0 & 0 & 3 \\
BBABBBDABA & 7 & 1 & 0 & 0 & 0 & 1 \\
BBACBBDACA & 8 & 0 & 1 & 0 & 0 & 1 \\
ABBAAABBAB & 9 & 0 & 0 & 0 & 1 & 1 \\
BBCBBBDACA & 10 & 0 & 0 & 1 & 0 & 1 \\
$\mathrm{~N}^{\mathrm{a}}$ & & 42 & 33 & 94 & 31 & 200 \\
No. haplotypes $^{\mathrm{b}}$ & & 6 & 4 & 4 & 2 & 10 \\
$\hat{G}^{\mathrm{c}}$ & & 1.592 & 1.458 & 1.190 & 1.067 & 1.284 \\
$H^{\mathrm{d}}$ & & 0.078 & 0.094 & 0.054 & 0.056 & 0.067 \\
\hline
\end{tabular}

${ }^{\text {a }}$ Total number of isolates included in the analyses for each (sub)population.

${ }^{\mathrm{b}}$ Number of unique haplotypes in each (sub)population.

${ }^{\mathrm{c}}$ Genotypic diversity calculated as $\hat{G}=1 / \sum\left[\mathrm{fx}(\mathrm{x} / \mathrm{N})^{2}\right]$ where $\mathrm{N}$ is the sample size and $\mathrm{fx}$ is the number of haplotypes occurring $\mathrm{x}$ times in the sample (Stoddart \& Taylor 1988).

${ }^{\mathrm{d}}$ Nei's genetic diversity (Nei 1973)

derived from the AFLP data. The fact that the AFLP data also show no evidence for random mating supports the assumption that the highly clonal data observed for the microsatellite data were not due to linkage of the microsatellite markers.

\section{Haplotype network}

The network constructed (Fig. 1) shows the phylogenetic relationships among the 10 haplotypes. Additional intermediate haplotypes were inferred where they were needed to connect the observed haplotypes by the most parsimonious paths. These intermediates were not found in the field survey. The distances along links between haplotypes are proportional to the minimum number of mutations required to form one haplotype from the other. The network has three informative features. There is a constellation consisting of closely related haplotypes differing by, at most, two mutations. Then there is a long tail representing seven mutations from the compact constellation to a single very distinct haplotype. There are also a number of short loops indicating alternative routes for the production of several of the haplotypes.

\section{Discussion}

There is extensive evidence that populations of $C$. parasiticum from diseased peanut plants in Georgia are clonal. We observed very low levels of genotypic diversity and widespread occurrence of a single haplotype. There was no significant population differentiation between sub-populations. No evidence of random mating was observed, even when using two independent marker systems (microsatellites and AFLP). Altogether, these results suggest that the fungus in Georgia is highly clonal throughout the region.

The haplotype network indicates the very close relationship of the overwhelming majority of all the isolates collected, once again inferring clonality. Most of them could have arisen by single mutations of 
other haplotypes that were also collected, or by simple recombinations with similar haplotypes. The simple loops likewise suggest convergent mutations or recombinations (Milgroom et al. 2008; Posada and Crandall 2001). The only exception to this pattern is

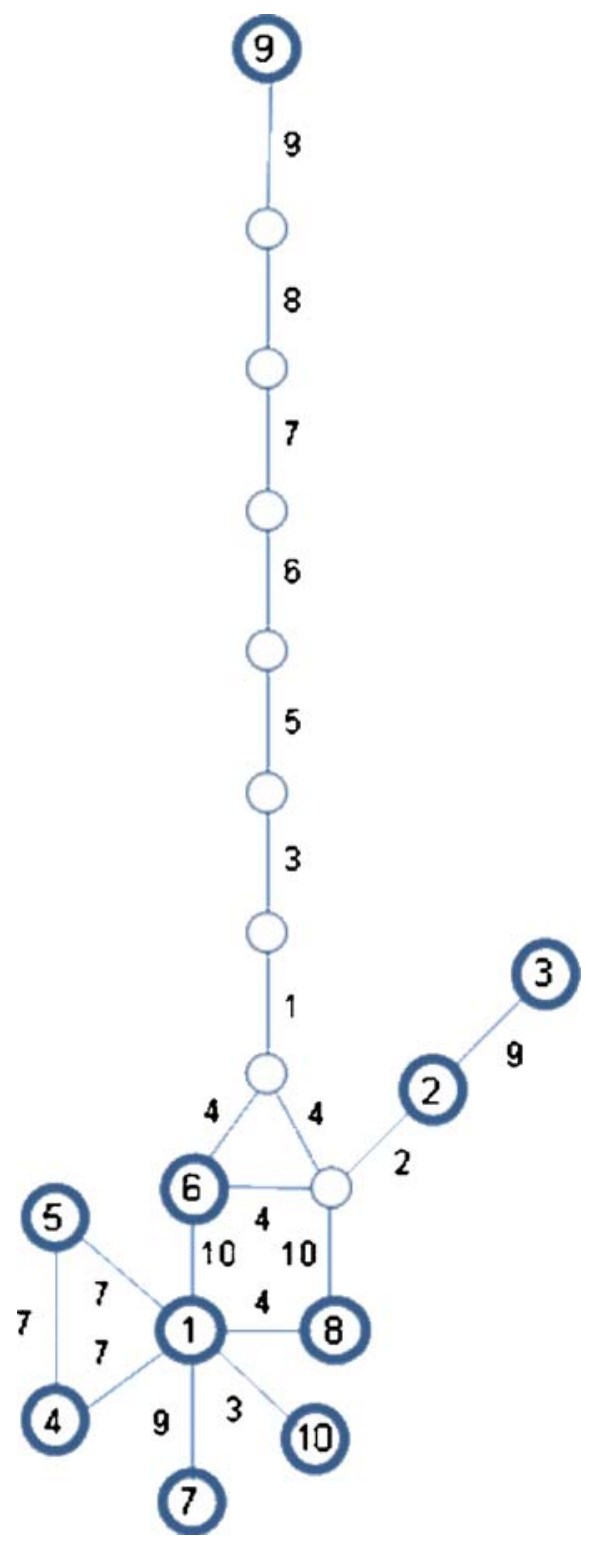

Fig. 1 Haplotype network for the fungal isolates obtained. The 10 individual multilocus haplotypes (MLH) are indicated by the numbers in nodes with heavy borders. Light-bordered nodes indicate the haplotypes on the most parsimonious route between the haplotypes obtained. None of these haplotypes was isolated in the field. Numbers next to the links between nodes indicate the single mutations required on the most parsimonious routes between haplotypes
MLH no. 9, a single isolate collected in the GA3 subpopulation. This isolate is so distinct that it is very unlikely to have been produced by mutation or recombination from any of the majority haplotypes. It is thus better seen as a rare immigrant to the area.

The haplotype network does not allow us to distinguish between clonality and sexual reproduction. The tight constellation of closely-related haplotypes could have been produced clonally, or by sexual reproduction that is either very localised mating or panmixis in a population of closely similar haplotypes. The network data do not allow us to determine the mating structure, however. In C. parasiticum mating is known to be very local and the population throughout Georgia is composed of very similar haplotypes. Both these, as well as clonality, could produce the network for our isolates. Thus it is not possible, in spite of the clonality, to exclude the possibility of recombination taking place between similar haplotypes.

Cylindrocladium parasiticum is a soil-borne pathogen that can spread via the movement of soil and plant debris infested with microsclerotia (Rowe et al. 1974; Krigsvold et al. 1977). Although ascospores are produced, their extreme susceptibility to lethal desiccation and the viscous ooze in which they are discharged limit their spread to short-distance within-field dispersal (Rowe and Beute 1975). The widespread occurrence of a single clone, however, suggests that seed transmission may be the primary mode of spread as suggested by Randall-Schadel et al. (2001) and Glenn et al. (2003). Seed transmission could also explain the rapid spread of the disease across the entire southeastern USA.

McDonald and Linde (2002) emphasise that knowledge of the population genetic structure of a pathogen could give an idea of its potential to overcome genetic resistance. Our results show that C. parasiticum in Georgia has a clonal genetic structure with no differentiation between populations. This means that recombination will be uncommon and mutations conferring the ability to break genetic resistance in the host will be unable to spread easily. In these terms at least, the disease can be seen as a low risk. On the other hand, the detection of a single isolate showing strong evidence of being introduced suggests that establishment of new haplotypes may be a significant threat. Furthermore, the possibility of recombination between the existing population and 
newly introduced haplotypes cannot be excluded. This emphasises the need to minimise the spread of C. parasiticum haplotypes, which is believed to take place principally through human agency and mainly through transport on seed.

This study represents a first attempt to understand the genetic variation within and among populations of C. parasiticum causing CBR in peanuts. The results demonstrate that microsatellite markers can be used effectively to analyse genotypic and genetic diversity of populations of $C$. parasiticum. These analyses can give rise to valuable information regarding the epidemiology of CBR, managing the disease and planning breeding strategies.

Acknowledgements We thank the National Research Foundation (NRF), members of the Tree Protection Co-operative Programme (TPCP) and the THRIP initiative of the Department of Trade and Industry (DTI), South Africa for financial support that made this study possible. We are also grateful to Almuth Hammerbacher for technical assistance.

\section{References}

Agapow, P.-M., \& Burt, A. (2001). Indices of multilocus linkage disequilibrium. Molecular Ecology Notes, 1, 101102.

Alfieri, S. A., El-Gholl, N. E., \& Schoulties, C. L. (1982). Homothallism in Calonectria ilicicola. Mycologia, 74, 513-514.

Bandelt, H.-J., Forster, P., \& Röhl, A. (1999). Median-joining networks for inferring intraspecific phylogenies. Molecular Biology and Evolution, 16, 37-48.

Bell, D. K., \& Sobers, E. K. (1966). A peg, pod, and root necrosis of peanuts caused by a species of Calonectria. Phytopathology, 56, 1361-1364.

Bell, D. K., Locke, B. J., \& Thompson, S. S. (1973). The status of Cylindrocladium black rot of peanut in Georgia since its discovery in 1965. Plant Disease Reporter, 57, 90-94.

Brenneman, T. (2003). Use of fumigation and resistant cultivars to manage Cylindrocladium black rot (CBR). In: T. B. Brenneman, \& C. L. Butts (Eds.), 2003 Georgia peanut research-extension report (pp. 76-82). Dawson: USDA. Retrieved November 20, 2008, from http://www.ars.usda.gov/sp2UserFiles/Place/66040000/ ResearchandExtensionReports/2003GAReport.pdf.

Crous, P. W., Wingfield, M. J., \& Alfenas, A. C. (1993). Cylindrocladium parasiticum sp. nov., a new name for $C$. crotalariae. Mycological Research, 97, 889-896.

Glenn, D. L., Phipps, P. M., \& Stipes, R. J. (2003). Incidence and survival of Cylindrocladium parasiticum in peanut seed. Plant Disease, 87, 867-871.
Hood, G. (2008). PopTools version 3.0.6. Retrieved February, 2009, from PopTools, CSIRO Australia Web site: http:// www.cse.csiro.au/poptools/download.htm.

Krigsvold, D. T., Garren, K. H., \& Griffin, G. J. (1977). Importance of peanut field cultivation and soybean cropping in the spread of Cylindrocladium crotalariae within and among peanut fields. Plant Disease Reporter, 61, 495-499.

McDonald, B. A., \& Linde, C. (2002). Pathogen population genetics, evolutionary potential, and durable resistance. Annual Review of Phytopathology, 40, 349-379.

Milgroom, M. G. (1996). Recombination and the multilocus structure of fungal populations. Annual Review of Phytopathology, 34, 457-477.

Milgroom, M. G., \& Peever, T. L. (2003). Population biology of plant pathogens: the synthesis of plant disease epidemiology and population genetics. Plant Disease, 87, 608-617.

Milgroom, M. G., Sotirovski, K., Spica, D., Davis, J. E., Brewer, M. T., Milev, M., et al. (2008). Clonal population structure of the chestnut blight fungus in expanding ranges in southeastern Europe. Molecular Ecology, 17, 4446-4458.

Möller, E. M., Bahnweg, G., \& Geiger, H. H. (1992). A simple and efficient protocol for isolation of high molecular weight DNA from filamentous fungi, fruit bodies, and infected plant tissues. Nucleic Acids Research, 20, 6115-6116.

Nei, M. (1973). Analysis of gene diversity in subdivided populations. Proceedings of the National Academy of Science of the United States of America, 70, 3321-3323.

Posada, D., \& Crandall, K. A. (2001). Intraspecific gene genealogies: trees grafting into networks. Trends in Ecology \& Evolution, 16, 37-45.

Randall-Schadel, B. L., Bailey, J. E., \& Beute, M. K. (2001). Seed transmission of Cylindrocladium parasiticum in peanut. Plant Disease, 85, 362-370.

Rowe, R. C., \& Beute, M. K. (1975). Ascospore formation and discharge by Calonectria crotalariae. Phytopathology, 65, 393-398.

Rowe, R. C., Johnston, S. A., \& Beute, M. K. (1974). Formation and dispersal of Cylindrocladium crotalariae microsclerotia in infected peanut roots. Phytopathology, 64, 1294-1297.

Stoddart, J. A., \& Taylor, J. F. (1988). Genotypic diversity: estimation and prediction in samples. Genetics, 118, 705-711.

Vos, P., Hogers, R., Bleeker, M., Reijans, M., \& van der Lee, T. (1995). AFLP: a new technique for DNA fingerprinting. Nucleic Acids Research, 23, 4407-4417.

Weir, B. S., \& Cockerham, C. C. (1984). Estimating F-statistics for the analysis of population structure. Evolution, 38, 1358-1370.

Wright, L. P., Wingfield, B. D., Crous, P. W., Brenneman, T., \& Wingfield, M. J. (2006). Isolation and characterization of microsatellite loci in Cylindrocladium parasiticum. Molecular Ecology Notes, 6, 110-112.

Yeh, F. C., Yang, R., \& Boyle, T. (1999). POPGENE Version 1.31: Microsoft window-based freeware for population genetic analysis. University of Alberta and Centre for International Forestry Research. 